\title{
The BH3-only protein Noxa is stimulated during apoptosis of chronic lymphocytic leukemia cells triggered by M2YN, a new plant-derived extract
}

\author{
MURHAF ZAHER $^{1-3}$, MASSOUD MIRSHAHI $^{1-4}$, YUSUF NURALIEV ${ }^{5}$, MIJGANA SHARIFOVA $^{5}$, \\ ISABELLE BOMBARDA ${ }^{6}$, JEAN-PIERRE MARIE ${ }^{1-3,7}$ and CHRISTIAN BILLARD ${ }^{1-3}$ \\ ${ }^{1}$ Centre de Recherche des Cordeliers, INSERM U872, Equipe $18 ;{ }^{2}$ Université Pierre et Marie Curie; \\ ${ }^{3}$ Université Paris-Descartes, UMRS 872, Paris, France; ${ }^{4}$ Academy of Sciences; ${ }^{5}$ International Institute \\ for Avicenna Heritage and Pharmacology, Dushanbe, Tajikistan; ${ }^{6}$ ISM2-AD2M, UMR CNRS 6263 , \\ Université Paul Cézanne, Marseille; ${ }^{7}$ Département d'Hématologie, Hôpital Saint-Antoine, Paris, France
}

Received March 22, 2011; Accepted May 12, 2011

DOI: 10.3892/ijo.2011.1121

\begin{abstract}
Deficiency of apoptosis is a hallmark of chronic lymphocytic leukemia (CLL) cells. M2Yn is a natural extract from plants of central Asia, identified for its antiangiogenic properties and its ability to block the migration of malignant cells. Here, we report that in vitro treatment of cells derived from CLL patients with M2Yn results in internucleosomal DNA fragmentation, phosphatidylserine externalization, mitochondrial membrane depolarization, caspase- 3 activation and cleavage of the caspase substrate PARP-1. The extents of these effects depend on the patients and are mostly comparable to those of flavopiridol or hyperforin, two known plant-derived apoptosis inducers of CLL cells. M2Yn does not modulate Mcl-1 expression, while downregulation of this antiapoptotic protein is involved in the action of flavopiridol. By contrast, M2Yn, like hyperforin, upregulates the Noxa protein, possibly by inhibiting proteasomal activity. This $\mathrm{BH} 3$-only protein is known to trigger the activation of the pro-apoptotic protein Bak through displacement of the Mcl-1/Bak complex at the mitochondrial membrane, as actually observed here in M2Yntreated cells. Our data, therefore, show that M2Yn can induce the caspase-dependent mitochondrial pathway of apoptosis in CLL cells via a mechanism resembling that of hyperforin. Our data also confirm that the BH3-only protein Noxa is a relevant target for CLL therapy.
\end{abstract}

\section{Introduction}

Chronic lymphocytic leukemia (CLL), the most frequent leukemia in Western countries (1), still remains an incurable

Correspondence to: Dr Christian Billard, UMRS 872, Equipe 18, Centre de Recherche des Cordeliers, 15 rue de l'Ecole de Médecine, 75006 Paris, France

E-mail: christian.billard@crc.jussieu.fr

Key words: M2Yn, apoptosis induction, CLL, Bcl-2 family, Noxa upregulation disease despite recent therapeutic advances with the combination of purine analogs, alkylating agents and monoclonal antibodies (2,3). CLL is characterized by clonal expansion of $\mathrm{CD}^{+} \mathrm{B}$ lymphocytes which accumulate in the blood and are deficient in their apoptotic program (4). Therefore, new combination treatment strategies based on the re-establishment of apoptosis and on newly identified molecular targets have to be developed $(2,4)$.

A number of agents are known for their ability to induce apoptosis of CLL cells in vitro, such as proteasome inhibitors, histone deacetylase (HDAC) inhibitors or various types of plant-derived compounds. Among them, flavopiridol, a semi-synthetic flavonoid, was the first to be discovered (5). Afterwards, we and others have shown the proapoptoptic properties of a number of natural products such as roscovitine, silvestrol, gossypol, resveratrol and other polyphenols, combretastatin analogues, some xanthones as well as hyperforin, a phloroglucinol purified from hypericum perforatum or $\mathrm{St}$ John's wort (6-11). Most of these agents, which often display antitumor and antiangiogenic properties, activate the caspasedependent mitochondrial pathway of apoptosis, but through different mechanisms. By its capacity to inhibit short-lived protein mRNA transcription, flavopiridol (and other cyclindependent kinase inhibitors) downregulates antiapoptotic proteins which are overexpressed in leukemic cells, including Mcl-1 (12,13), a Bcl-2 family member thought to be crucial for apoptosis deficiency in CLL (14). In contrast to flavopiridol, hyperforin does not inhibit Mcl-1 but induces the expression of the proapoptotic protein Noxa (15) belonging to the BH3-only subclass of the Bcl-2 family (16). Proteasome and HDAC inhibitors also upregulate Noxa $(17,18)$. However, clinical trials performed in CLL with apoptosis inducers turned out to be disappointing. This is the case for flavopiridol (19), bortezomib, a proteasome inhibitor (20) or HDAC inhibitors (21), while no clinical trial with hyperforin has been reported so far. It therefore appears necessary to identify new therapeutic agents capable of specifically targeting certain $\mathrm{Bcl}-2$ family proteins.

M2Yn is a natural extract isolated from plants in high altitude areas of central Asia which has been used in traditional 
medicine for centuries to alleviate symptoms associated with viral infections, particularly hepatitis-like diseases. M2YN was characterized in our laboratory for its potential antiangiogenic properties (Mirshahi et al, unpublished data). Actually, this compound inhibits the formation of vascular tubules by human bone marrow and microvascular endothelial cells in vitro. Moreover, it blocks the migration of invasive mammary carcinoma cells, as well as filopode and lamellipode formation by corneal fibroblasts via Rho protein modifications, which are critical events in cell migration and tumor invasiveness.

In the present work, we have investigated the effects of M2Yn on nuclear, membrane and mitochondrial events of apoptosis on CLL patients' cells ex vivo in comparison with flavopiridol and hyperforin, two apoptosis inducers of vegetal origin. We show evidence that M2Yn can trigger the caspasedependent mitochondrial pathway of apoptosis in the leukemic cells, involving upregulation of the proapoptotic Noxa without affecting the expression of the antiapoptotic Mcl-1. Our data also confirm that the $\mathrm{BH} 3$-only protein Noxa is an attractive target for new therapeutic strategies in CLL.

\section{Patients and methods}

Patients, cells and cell culture. Blood samples from CLL patients were obtained from the Hematology Department of Saint-Antoine Hospital (Paris, France) directed by one of us (Jean-Pierre Marie), after written informed consent in accordance with the revised Helsinki protocol. Diagnosis was established according to standard clinical criteria and to the International Workshop on CLL (IWCLL), including lymphocyte morphology and co-expression of CD5, CD20 and CD23 antigens (22). A total of 7 untreated patients (4 men and 3 women), all classified as Binet stage $\mathrm{A}$, with a mean age of $63 \pm 10$ years were selected. The patients were randomly chosen inasmuch as CD38 and ZAP-70 expression, cytogenetic data and mutational $\mathrm{VH}$ status were available only for some of them, thus hampering a risk-group analysis. Leukemic B cells were isolated from peripheral blood of CLL patients with a purity $\geq 95 \%$ as previously described (23) and were immediately cultured. Cell cultures were performed at $37^{\circ} \mathrm{C}$ in a humidified atmosphere containing $5 \% \mathrm{CO}_{2}$ in RPMI-1640 medium supplemented with $2 \mathrm{mM}$ glutamine, antibiotics and $10 \%$ FCS (PAA Laboratories, Pasching, Austria) at seeding densities of $2-4 \times 10^{6}$ cells $/ \mathrm{ml}$. Cell concentrations were measured with a Z2 Coulter counter (Beckman-Coulter, Les Ulis, France) and cell viability was evaluated using a colorimetric assay based on WST1 tetrazolium salt cleavage by mitochondrial dehydrogenases (Roche Diagnostic, Meylan, France).

Reagents. M2Yn was isolated from medicinal plants harvested in high altitude areas of Tajikistan. The plants including their propolis were dried and the resulting powder (100 g) was subjected to alcoholic extraction $(10 \% \mathrm{w} / \mathrm{v}$ in $70 \%$ ethanol). After filtration, the extract was dry-concentrated and samples were dissolved at the concentration of $3 \mathrm{mg} / \mathrm{ml}$ in $70 \%$ ethanol. The M2Yn extract thus obtained endows with antiangiogenic and antimigratory properties in vitro: it inhibits the formation of pseudo-tubules by endothelial cells grown on matrigel, decreases the motility of corneal fibroblasts and blocks the migration of invasive mammary carcinoma cells (Mirshahi et al, unpublished data). These biological activities are detectable with a concentration of $0.5 \mu \mathrm{g} / \mathrm{ml}$, reaching optimal values with $1-2 \mu \mathrm{g} / \mathrm{ml}$. M2Yn is much more active than different propolis of european origin. The partial chemical analysis of M2Yn extract revealed that it contains several flavonoids, tocopherols, polyunsaturated fatty acids and chlorophyll, and mass spectrometry showed the presence of several polypeptides unknown in protein data base. However, M2Yn retained its biological properties upon protein depletion by saturated ammonium sulfate. Flavopiridol was kindly provided by Aventis Pharmaceuticals (Bridgewater, NJ, USA). Hyperforin was purified by one of us (Isabelle Bombarda) in the laboratory which described the procedure (24). Most other reagents and chemicals were from Sigma (Sigma Aldrich, Saint Quentin Fallavier, France), except when otherwise stated.

Phosphatidylserine (PS) externalization. This membrane marker of apoptosis was quantified by specific binding of FITC-conjugated annexin V (Bender Medsystems, Vienna, Austria) with or without simultaneous labeling with propidium iodide (PI), as previously detailed (23). The percentages of cells positive for annexin V-FITC and PI negative (early apoptosis) and of double positive cells (late apoptosis) were determined with a flow cytometer (Beckman Coulter).

Internucleosomal DNA fragmentation. The cleavage of chromatin and DNA into nucleosomes, a typical nuclear event of apoptosis, was evaluated by detection of cytoplasmic histoneassociated DNA fragments (mono- and oligonucleosomes) in cell lysates from aliquots of 20,000 cells, using an ELISA with anti-histones and anti-DNA fragments mAbs (Cell Death Detection ELISAPLUS, Roche Diagnostics) according to a technique used previously (23). Nucleosomes levels were measured using streptavidin/biotin/peroxidase system and reading of the absorbance at $405 \mathrm{~nm}$. Values obtained from triplicate samples never differed more than $10 \%$.

Mitochondrial membrane depolarization. Activation of the mitochondrial pathway of apoptosis leads to mitochondrial membrane permeabilization characterized by a dissipation of the membrane potential. This depolarization can be quantified by a shift of the red to the green fluorescence of the specific probe JC-1, and therefore by a decrease of red/green fluorescence ratio, according to a technique described previously (23). Briefly, $2 \times 10^{6}$ cells $/ \mathrm{ml}$ were incubated in an assay buffer containing $2 \mu \mathrm{M} \mathrm{JC}-1$ for $15 \mathrm{~min}$ at $37^{\circ} \mathrm{C}$, then washed and distributed in a microtitration plate. Green and red fluorescences (respectively: excitation wavelength $485 \mathrm{~nm} / \mathrm{emission}$ wavelength $525 \mathrm{~nm}$, and excitation wavelength $485 \mathrm{~nm} /$ emission wavelength $595 \mathrm{~nm}$ ) were simultaneously recorded with a microplate reader cytofluorometer (Wallac Victor-2, Perkin-Elmer, Norwalk, MT, USA).

Caspase-3 activity assay. Enzymatic activity of caspase-3 was determined by the capacity of cell lysates to cleave a specific substrate according to techniques previously used (23). Briefly, aliquots of cell lysates (10-20 $\mu \mathrm{g})$ were incubated with the fluorogenic caspase-3 substrate DEVD-aminocoumarin 
(DEVD-AMC) and the release of AMC was then recorded every $10 \mathrm{~min}$ for $2 \mathrm{~h}$ with a Victor- 2 thermostated at $37^{\circ} \mathrm{C}$ (excitation wavelength: $380 \mathrm{~nm} /$ emission wavelength: $460 \mathrm{~nm}$ ). The specificity of the reaction was assessed by the addition of unlabeled Ac-DEVD-CHO caspase-3 inhibitor (Sigma-Aldrich).

Western blotting. Protein expression was studied by Western blotting, as detailed previously (23). Equal amounts of the cell lysates (30-50 $\mu \mathrm{g}$ of proteins) were electrophoresed by SDS-PAGE. Separated proteins were electrotransferred on PVDF membranes which were further incubated with primary antibodies recognizing the molecules of interest. The immunoblotted proteins were revealed with secondary HRP-specific antibodies (DakoCymation, Glostrup, Denmark) and a system of enhanced chemiluminescence (Amersham ECL Western Blotting Analysis System, GE Healthcare, Orsay, France). The primary antibodies used were: the rabbit polyclonal S-19 anti-Mcl-1 and the mouse mAb anti-Bcl-2 (both from Santa Cruz Biotechnology, Tebu, Le Perray en Yvelines, France); two mouse mAb anti-Noxa, clone 6D619 (US Biologicals, Euromedex, Mundolsheim, France) and IMG-249 (Imgenex, Clinisciences, Montrouge, France); the mouse mAb anti-Bak Ab-1 specific for the active form of Bak (Calbiochem, VWR International SAS, Fontenay, France); the mouse $\mathrm{mAb}$ antiPARP-1 [Poly(ADP-ribose) Polymerase-1] recognizing both the native form of $116 \mathrm{kDa}$ and the cleaved fragment $85 \mathrm{kDa}$ (Santa Cruz Biotechnology); the rabbit polyclonal anti-p27 (N20) recognizing both the native form $(27 \mathrm{kDa})$ and the cleaved fragment p23 (Santa Cruz Biotechnology); the antibeta actin (clone C4; MP Biomedicals, Illkirch, France) used for protein content monitoring and standardization.

Immunoprecipitation experiments. Cells were lysed for $30 \mathrm{~min}$ at $4^{\circ} \mathrm{C}$ with $1 \% 3$-[(3-cholamidopropyl)dimethylammonio]-1propane sulfonate (CHAPS), $20 \mathrm{mM}$ Tris-HCl/pH 8.0, $150 \mathrm{mM}$ $\mathrm{NaCl}$ and a cocktail of protease inhibitors. Cell lysates were pre-cleared for $30 \mathrm{~min}$ at $4^{\circ} \mathrm{C}$ with Protein G-agarose beads (Santa Cruz Biotechnology) equilibrated with lysis buffer in the presence of the isotype antibody. Pre-cleared lysates were then incubated overnight at $4^{\circ} \mathrm{C}$ with pre-equilibrated Protein $\mathrm{G}$ beads and the mouse mAb anti-Mcl-1 (MCL-1801) from Santa Cruz Biotechnology. Immunoprecipitates were washed and mixed with non-reducing electrophoresis sample buffer, and beads were discarded by boiling for $3 \mathrm{~min}$. Immunoprecipitates were finally subjected to Western blot analysis using the following primary antibodies: either anti-Mcl-1 (S-19) as a control of IP, anti-Noxa (6D619) or the rabbit polyclonal anti-Bak NT (Upstate, Millipore, Saint Quentin en Yvelines, France).

Immunofluorescence and flow cytometry analysis. The expression of Noxa protein was also analyzed by indirect immunofluorescence on cells permeabilized at $4^{\circ} \mathrm{C}$ with the Cytofix/Cytoperm kit (BD Biosciences, Le Pont de Claix, France), as previously described (23). Cells were incubated with for $30 \mathrm{~min}$ with primary antibodies: either goat polyclonal anti-Noxa (N15, Santa Cruz Biotechnology) or isotype control (goat $\mathrm{IgG}$ ). Cells were further incubated with a secondary FITC-conjugated antibody against goat Ig before to be analyzed by flow cytometry using an EPICS Altra flow cytometer (Beckman-Coulter) in order to record the profiles of fluorescence and the percentages of fluorescent cells.

RT-PCR analysis of mRNA expression. Total RNA was extracted from CLL cells with the Trizol method and reverse transcription was performed as previously detailed (25). The primers used for Noxa and Mcl-1 (synthesized Eurobio, Les Ulis, France) and for $\beta 2$-microglobulin (Sigma Proligo France) were as follows: Noxa (forward) 5'-GTG CCC TTG GAA ACG GAA GA-3', and (reverse) 5'-CCA GCC GCC CAG TCT AAT CA-3'; Mcl-1 (forward) 5'-ATC TCT CGG TAC CTT CGG GAG C-3', and (reverse) 5'-CCT GAT GCC ACC TTC TAG GTC C-3'; $\beta 2$-microglobulin (forward) 5'-CAT CCA GCG TAC TCC AAA GA-3', and (reverse) 5'-GAC AAG TCT GAA TGC TCC A-3'. The annealing temperature of primers was $57^{\circ} \mathrm{C}$. PCR products were visualized by electrophoresis in $2 \%$ agarose gel containing $0.2 \mathrm{mg} / \mathrm{ml}$ ethidium bromide. The sizes of amplified cDNAs were $165 \mathrm{bp}$ for $\beta 2$-microglobulin, 446 bp for Mcl-1, and 260 bp for Noxa.

Proteasome activity assay. Proteasome activity was determined with a luminescent assay, the Proteasome-Glo ${ }^{\mathrm{TM}}$ Chymotrypsin-like cell-based assay (Promega France, Charbonnières-les-Bains, France) that measures chymotrypsin-like protease activity associated with the proteasome complex directly in intact cells. This assay has been used to evaluate the activity of bortezomib in a variety of cancer cell lines (26). It has three components: a buffer containing the proteasome complex, a luciferin detection reagent and the luminogenic substrate, succinyl-LLVY-aminoluciferin. The proteasome cleavage generates a luminescent signal produced by the luciferase reaction. Briefly, $100 \mu \mathrm{l}$ of the reagent mixture was added to $2 \times 10^{5}$ cells $/ 100 \mu \mathrm{l}$ in 96 -well microtitration plates for $15 \mathrm{~min}$ at $37^{\circ} \mathrm{C}$, and the luminescence was immediately recorded with a spectrophotometer Wallac Victor-2 microplate reader.

\section{Results}

M2Yn induces apoptosis of primary CLL cells. To investigate the effects of M2Yn on internucleosomal DNA fragmentation in CLL cells from different patients, cells were treated in culture with various M2Yn concentrations ranging from 0.5 to $12 \mu \mathrm{g} / \mathrm{ml}$ for different periods of time not exceeding $48 \mathrm{~h}$ in order to prevent spontaneous apoptosis in the untreated controls. It turned out that M2Yn treatment resulted in a time- and dose-dependent stimulation of DNA fragmentation, and Fig. 1 shows the maximal effect observed after $18 \mathrm{~h}$ with $6 \mu \mathrm{g} / \mathrm{ml}$ in comparison with $1 \mu \mathrm{M}$ flavopiridol and $10 \mu \mathrm{g} / \mathrm{ml}$ hyperforin. The amplitude of this effect of M2Yn differed depending on the patients but was comparable to the effects of flavopiridol and hyperforin: a 3.9-fold mean increase over untreated controls was found with M2Yn versus 4.1- and 5.1-fold with respectively flavopiridol and hyperforin. M2Yn treatment also stimulated PS externalization as illustrated in Table I: the percentages of cells positive for PS externalization markedly augmented when compared to untreated controls. These data indicate that M2Yn is capable of inducing the apoptosis of CLL cells in vitro. 


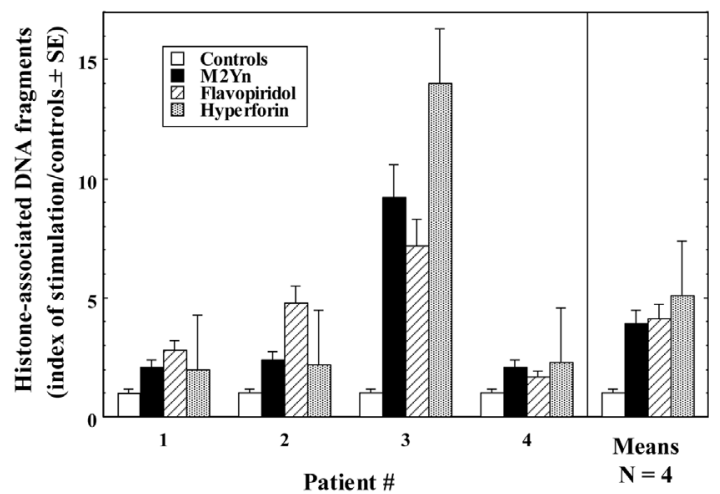

Figure 1. Effect of M2Yn on internucleosomal DNA fragmentation in CLL patient cells and comparison with flavopiridol and hyperforin. Leukemic cells from 4 CLL patients were cultured for $18 \mathrm{~h}$ in the absence (controls) or the presence of either $6 \mu \mathrm{g} / \mathrm{ml} \mathrm{M} 2 \mathrm{Yn}, 1 \mu \mathrm{M}$ flavopiridol, or $10 \mu \mathrm{g} / \mathrm{ml}(18 \mu \mathrm{M})$ hyperforin, then cytoplasmic histone-associated DNA fragments (nucleosomes) were measured as described in Materials and methods.

M2Yn-induced apoptosis involves the caspase-dependent mitochondrial pathway. To examine whether the apoptosis elicited by M2Yn was caspase-dependent, the enzymatic activity of the effector caspase- 3 was analyzed by measuring the capacity of cell lysates to cleave a specific substrate. Results showed that caspase-3 activity was stimulated after exposure of CLL patient cells to M2Yn. The examples of two patients are presented in Fig. 2A where caspase-3 activity was enhanced by M2Yn to the same extent as flavopiridol or hyperforin in one patient, but to a lesser extent in another one. Furthermore, the release of apoptogenic factors responsible for the cascade of caspase activation is allowed by pore opening at the mitochondrial membrane. This is associated with a dissipation of the transmembrane potential. It clearly appears that M2Yn treatment of CLL cells resulted in the diminution of the mitochondrial membrane potential, as shown in Fig. 2B. The amplitude of this effect depended on individual patient and was comparable to that obtained with flavopiridol or hyperforin. These results indicate that M2Yn triggers the caspase-dependent mitochondrial pathway of apoptosis in CLL cells.

M2Yn treatment stimulates Noxa expression in CLL cells. We then analyzed the effects of M2Yn on the expression of three Bcl-2 family proteins regulating the mitochondrial pathway of apoptosis: the proapototic BH3-only Noxa known to be upregulated during hyperforin-induced apoptosis (12), and two antiapoptotic proteins which are overexpressed by CLL cells and downregulated by flavopiridol, Bcl-2 and the crucial Mcl-1 (12,23). Apoptosis induction was monitored in parallel by the cleavages of PARP-1, a substrate of caspases, and of $\mathrm{p} 27^{\mathrm{kip} 1}$ which is overexpressed by CLL cells and downregulated during apoptosis triggered by flavopiridol or hyperforin through caspase-dependent cleavage (23). Western blotting obtained from four patients are presented in Fig. 3A. As expected (15), flavopiridol-induced PARP-1 and p27 cleavages were associated with a strong Mcl-1 inhibition, a slight Bcl-2 cleavage and no stimulatory effect on Noxa levels. As also expected (15), hyperforin elicited PARP-1, p27 ${ }^{\mathrm{kip} 1}$ and Bcl-2 cleavages to a lesser extent than flavopiridol, did not inhibit
Table I. Effects of M2Yn on PS externalization in CLL cells in comparison with flavopiridol and hyperforin.

Percentages of cells

\begin{tabular}{lccccc}
\cline { 2 - 6 } & $\mathrm{A}^{-} / \mathrm{PI}^{-}$ & $\mathrm{PI}^{+}$ & $\mathrm{A}^{+}$ & $\mathrm{A}^{+} / \mathrm{PI}^{+}$ & Total A $^{+}$ \\
\hline Controls & 62 & 4 & 11 & 22 & 33 \\
M2Yn & 36 & 2 & 35 & 26 & 61 \\
Flavopiridol & 11 & 1 & 72 & 15 & 87 \\
Hyperforin & 18 & 9 & 23 & 49 & 72 \\
\hline
\end{tabular}

CLL cells from patient no. 5 were treated for $42 \mathrm{~h}$ with $6 \mu \mathrm{g} / \mathrm{ml}$ $\mathrm{M} 2 \mathrm{YN}, 1 \mu \mathrm{M}$ flavopiridol, or $20 \mu \mathrm{g} / \mathrm{ml}$ hyperforin, or not treated (controls). PS externalization was evaluated by labeling with FITCannexinV/PI and flow cytometry analysis, as described in Materials and methods. Data are from one experiment representative of three. $\mathrm{A}^{-} / \mathrm{PI}^{-}$, double negative viable cells; $\mathrm{PI}^{+}$, PI positive necrotic cells; $\mathrm{A}^{+}$, annexin $\mathrm{V}$ positive cells (early apoptosis); $\mathrm{A}^{+/} \mathrm{PI}^{+}$: double positive cells (late apoptosis); Total $\mathrm{A}^{+}$, apoptotic cells.

Mcl-1 but strongly enhanced Noxa levels. It turned out that treatment with M2Yn resulted in the stimulation of Noxa expression versus untreated controls, with hardly detectable effects on Mcl-1 levels or Bcl-2 cleavage. Mcl-1 expression was nearly not affected even with high concentrations of M2Yn (data not shown). M2Yn-promoted Noxa stimulation was dose-dependent and was accompanied with PARP-1 and p27 cleavages to extent similar to those observed with hyperforin. The effect of M2Yn on Noxa expression was not as strong as that of the phloroglucinol, but was observed with all the patients in the study (7/7). Moreover, it was obtained with two different anti-Noxa antibodies and was also detected by immunofluorescence (with a third antibody) and flow cytometry analysis: indeed both fluorescence intensity and the percentage of positive cells increased after treatment with M2Yn (Fig. 3B). These results show that unlike flavopiridol, but similarly to hyperforin, M2Yn induces Noxa upregulation without detectable effect on Mcl-1 expression and that this effect is associated with apoptosis.

M2Yn does not increase Noxa mRNA expression but inhibits proteasome activity. To evaluate whether the effect of M2Yn on Noxa protein resulted from transcription activation, RT-PCR experiments were performed. Fig. 4A shows that Noxa mRNA levels remained unchanged after treatment of CLL cells with M2Yn. No effect on Mcl-1 mRNA levels was observed while these levels decreased after treatment with flavopiridol, in agreement with previous data (13). These results indicate that the stimulatory effect of M2Yn on Noxa protein does not seem to result from a transcriptional regulation.

Because Noxa is known to be upregulated by inhibition of proteasomal degradation $(16,17,27)$ and because a number of plant-derived compounds are natural proteasome inhibitors (28), we decided to study the effect of M2Yn on proteasomal activity in CLL cells. As seen in Fig. 4B, M2Yn treatment induced a dose-dependent reduction in proteasomal activity 

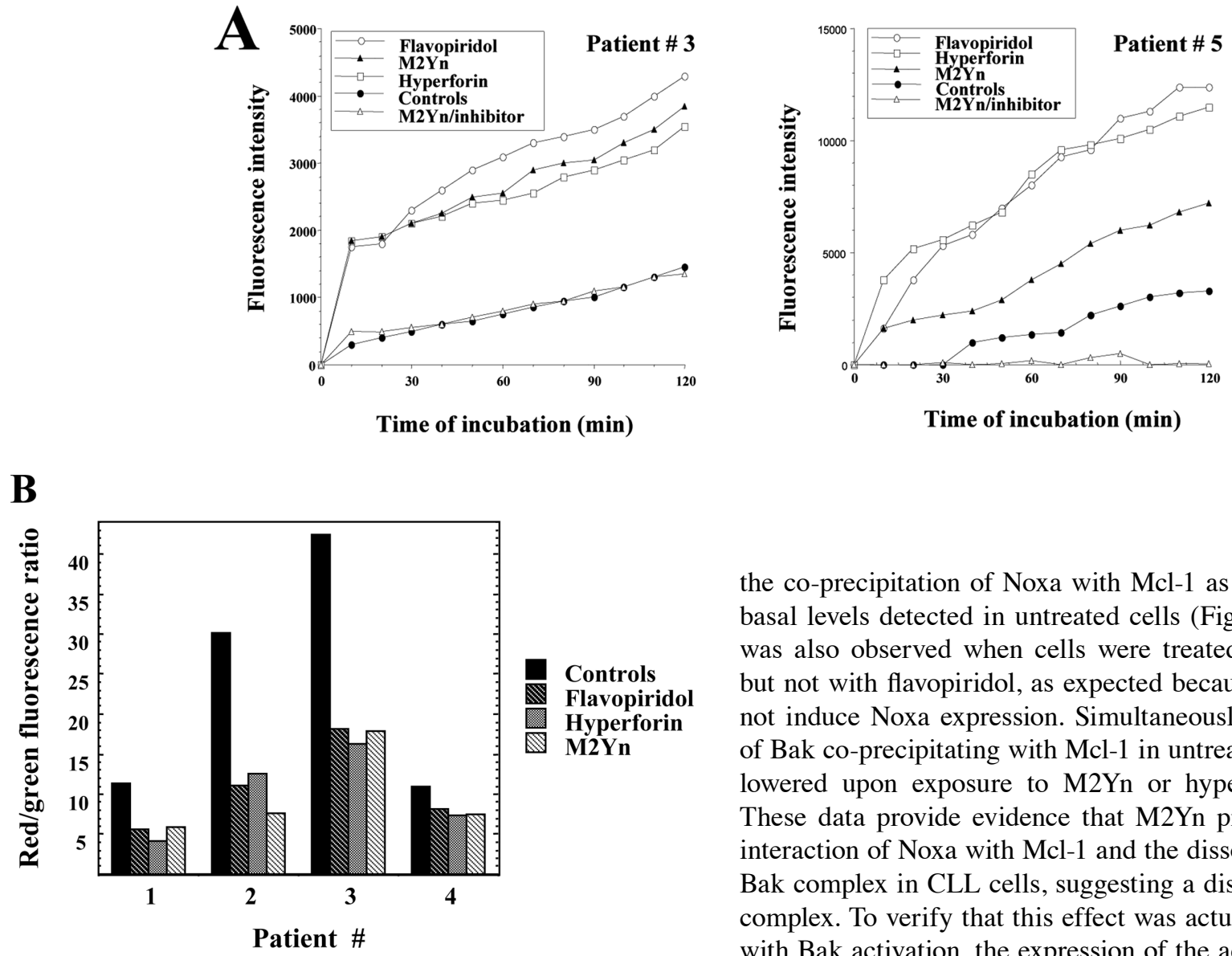

Figure 2. Effects of M2Yn on caspase-3 activity and on the mitonchondrial membrane potential in comparison with flavopiridol and hyperforin. Cells from different CLL patients were treated with either $6 \mu \mathrm{g} / \mathrm{ml} \mathrm{M} 2 \mathrm{Yn}, 1 \mu \mathrm{M}$ flavopiridol, or $10 \mu \mathrm{g} / \mathrm{ml}$ hyperforin, or left untreated (controls), then analyzed for caspase-3 activity and variation of mitochondrial membrane potential. (A) M2Yn stimulates caspase-3 activity, as measured by the capacity of cell lysates to cleave the fluorogenic specific substrate DEVD-AMC every 10 min during $2 \mathrm{~h}$; the specificity of the enzymatic reaction was monitored by simultaneous incubation of the cell lysates with an inhibitor of caspase-3 activity (DEVD-CHO), as exemplified with M2Yn-treated cells (M2Yn/ inhibitor). (B) M2Yn induces a dissipation of the mitochondrial membrane potential, as revealed by a decrease in the red/green fluorescence ratio using the specific probe JC-1. Data are means of triplicate values with SD never exceeding $10 \%$. See Materials and methods for experimental procedures.

even more efficiently than hyperforin, although to a lower extent than bortezomib, a proteasome inhibitor used as a reference. It is thus possible that such a post-translational regulation might contribute to M2Yn-promoted Noxa upregulation.

M2Yn stimulates Mcl-1/Noxa association, Mcl-1/Bak dissociation and the active form of Bak. Noxa is known to insert its BH3 domain into the hydrophobic groove of Mcl-1 resulting in the displacement of the complex that Mcl-1 forms with the proapototic Bcl-2 family protein Bak, and thus in Bak release and activation (16). We therefore investigated whether Noxa, when upregulated by M2Yn, can interact with Mcl-1 and displace Mcl-1/Bak complex. To this end, co-immunoprecipitation experiments were performed on CLL cell lysates immunoprecipitated by an anti-Mcl-1 antibody. Results show clearly that treatment with M2Yn stimulated

the co-precipitation of Noxa with Mcl-1 as compared to low basal levels detected in untreated cells (Fig. 5A). This effect was also observed when cells were treated with hyperforin but not with flavopiridol, as expected because the latter does not induce Noxa expression. Simultaneously, the high levels of Bak co-precipitating with Mcl-1 in untreated controls were lowered upon exposure to M2Yn or hyperforin (Fig. 5A). These data provide evidence that M2Yn promoted both the interaction of Noxa with Mcl-1 and the dissociation of Mcl-1/ Bak complex in CLL cells, suggesting a displacement of this complex. To verify that this effect was actually accompanied with Bak activation, the expression of the active form of Bak was studied. As seen in Fig. 5B, treatment of CLL cells with M2Yn resulted in a concentration-dependent stimulation of activated Bak levels, with a maximal extent closely resembling the effect of hyperforin. It can be noted that a similar result was observed upon exposure to flavopiridol that does not induce Noxa upregulation but Mcl-1 downregulation.

\section{Discussion}

The identification of new agents having highly specific targets is necessary to improve CLL therapy. Proteins of the Bcl-2 family appear as potential targets because they regulate survival/apoptosis balance via the control of pore opening at the mitochondrial membrane, allowing the release of apoptogenic factors (16). Moreover, this delicate balance influences CLL cell fate (29). Several reports have recently revealed the crucial role of Mcl-1 in the apoptotic defect of CLL cells $(14,30)$. Besides, loss of Mcl-1 is sufficient by itself to trigger programmed cell death (31). Due to its specific interaction with Mcl-1, the BH3-only Noxa seems also to be an interesting target for CLL therapy $(15,16,32)$. Actually, Noxa antagonizes Mcl-1, thus facilitating the activation of Bak, responsible for the mitochondrial membrane permeabilization and the subsequent caspase cascade.

In the present study, we show that the natural extract M2Yn, like a number of antitumor/antiangiogenic compounds of vegetal origin (flavopiridol, resveratrol and other polyphenols, hyperforin), is capable of inducing the apoptosis of primary CLL cells. As most apoptosis inducers, M2Yn activates the caspase-dependent mitochondrial pathway. This is attested by stimulation of DNA fragmentation, PS external- 


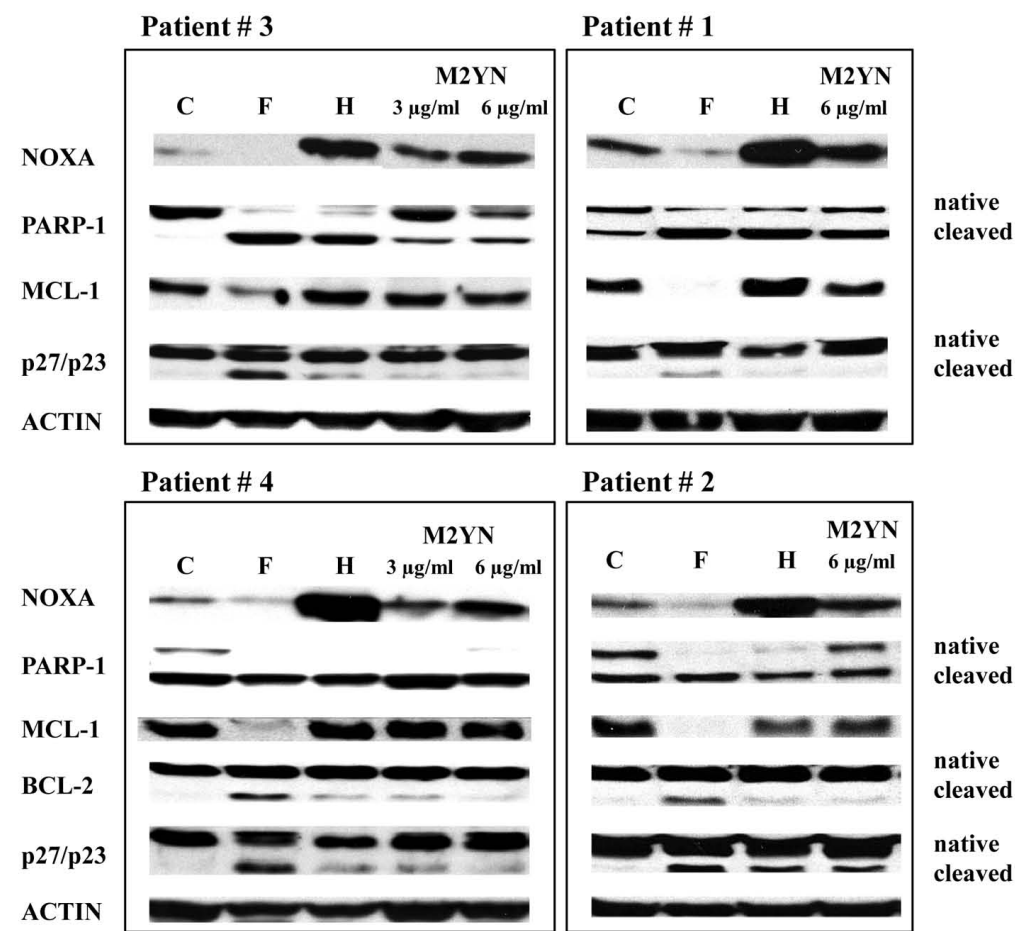

B

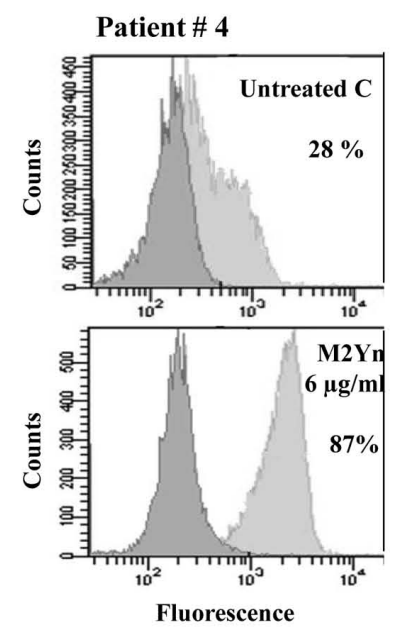

eaved

ative

(1)

Figure 3. M2Yn stimulates Noxa protein expression in CLL cells. CLL cells of patients were treated for $18 \mathrm{~h}$ with either 3 or $6 \mu \mathrm{g} / \mathrm{ml} \mathrm{M} 2 \mathrm{Yn}, 1 \mu \mathrm{M}$ flavopiridol (F) or $10 \mu \mathrm{g} / \mathrm{ml}$ hyperforin (H) and in untreated controls (C). (A) Western blot analysis of the expression and/or cleavage of different proteins showing that M2Yn stimulates both Noxa expression and PARP-1 cleavage without modulating Mcl-1 levels. (B) Immunofluorescence and flow cytometry analysis of Noxa expression in CLL cells from patient no. 4 after 18-h treatment with $6 \mu \mathrm{g} / \mathrm{ml}$ M2Yn and in untreated controls (C); the data represent the fluorescence profiles obtained with anti-Noxa antibody (pale grey) in comparison with those obtained with isotype control Ig (dark grey) and the percentages of positive cells are indicated.

A

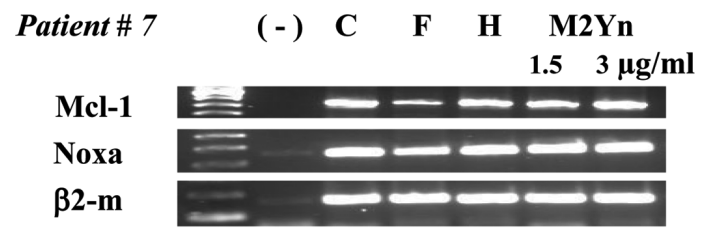

B

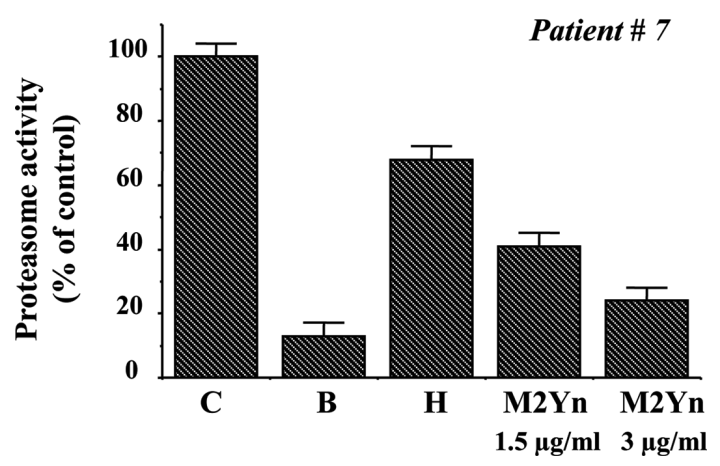

Figure 4. M2Yn treatment does not modulate Noxa mRNA expression but inhibits proteasomal activity in CLL cells. Cells from patients 3, 6 and 7 were cultured for $18 \mathrm{~h}$ in the presence of 1.5 or $3 \mu \mathrm{g} / \mathrm{ml} \mathrm{M} 2 \mathrm{Yn}, 1 \mu \mathrm{M}$ flavopiridol $(\mathrm{F}), 10 \mu \mathrm{g} / \mathrm{ml}$ hyperforin $(\mathrm{H})$, or $5 \mathrm{nM}$ bortezomib (B) or in their absence (controls, C). (A) Representative RT-PCR analysis of Noxa and Mcl-1 mRNA levels with $\beta 2-\mathrm{m}$ as a control of RNA content; (-): buffer without cDNA. (B) Dose-dependent inhibition of proteasomal activity by M2Yn (mean of duplicates in a representative experiment). ization, mitochondrial membrane permeabilization, caspase-3 activation as well as cleavage of the caspase substrate PARP-1. M2Yn has no direct effect on the expression of Mcl-1 but neutralizes its antiapoptotic activity through Noxa upregulation. Indeed, by interacting with Mcl-1, Noxa displaces Mcl-1/Bak complex leading to Bak activation. Consequently, the mechanism of action of M2Yn is different from that of flavopiridol and other plant-derived compounds acting through downregulation of antiapoptotic proteins including Mcl-1 $(12,13)$. By upregulating the proapoptotic Noxa, M2Yn employs a mechanism shared by hyperforin, proteasome and HDAC inhibitors $(15,17,18,32)$. This effect appears to result from a regulation at the protein level, as suggested by the ability of M2Yn to inhibit proteasomal activity in CLL cells, as do proteasome inhibitors (17) and hyperforin (Zaher et al, unpublished). With the exception of hyperforin which has not been so far tested therapeutically, apoptosis inducers of CLL cells such as flavopiridol, HDAC and proteasome inhibitors turned out to be disappointing in clinical trials, showing toxicity, adverse effects and/or no significant therapeutic activity (19-21). This is not surprising because their targets are not really specific: flavopiridol inhibits the transcription of a large number of proteins (33); HDAC and proteasome inhibitors enhance the expression of not only Noxa but also antiapoptotic proteins such as Mcl-1 $(34,35)$. The observation that M2Yn does not modulate Mcl-1 expression seems therefore of great interest. The characterization of the activity of 
A

Patient \# 3
Patient \# 6

\author{
IP: Mcl-1
}

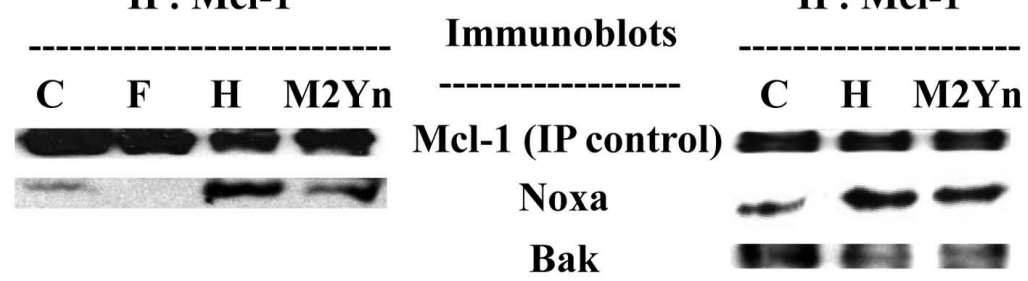

B

Patient \# 3

$\mu \mathrm{g} / \mathrm{ml}$ M2Yn

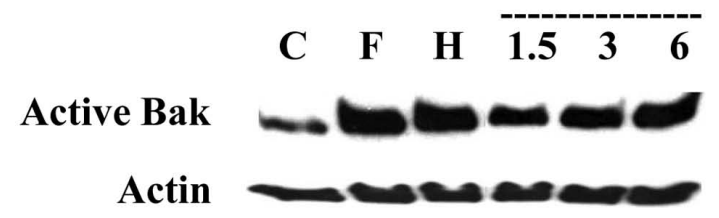

Figure 5. M2Yn stimulates Noxa/Mcl-1 association, Mcl-1/Bak dissociation and activated Bak expression. CLL cells from patients 3, 6 and 7 were treated for $18 \mathrm{~h}$ with either $1 \mu \mathrm{M}$ flavopiridol (F), $10 \mu \mathrm{g} / \mathrm{ml}$ hyperforin $(\mathrm{H})$ or $6 \mu \mathrm{g} / \mathrm{ml} \mathrm{M} 2 \mathrm{Yn}$, or left untreated (controls, C). (A) Representative co-immunoprecipitations experiments showing that M2Yn treatment increases the co-precipitation of Noxa in Mcl-1 immunoprecipitates (IP) and that it decreases the co-precipitation of Bak in Mcl-1 IP (right panel). (B) Representative Western blot analysis showing that the levels of the active form of Bak are dose-dependently stimulated by M2Yn after 18-h treatment.

M2Yn and of its mechanism of action suggests that it belongs to a possible class of plant-derived apoptosis inducers capable of specifically targeting the BH3-only protein Noxa through upregulation. This family of compounds includes hyperforin, a phloroglucinol, and the natural proteasome inhibitor celastrol, a triterpen which was reported to induce apoptosis by activating Noxa (27). It has to be noted that triterpenoids can induce apoptosis of CLL cells (36). It is possible that a number of flavonoids (quercetin, apigenin, myricetin) known for their capacity to inhibit proteasome activity (28) would be able to induce apoptosis by enhancing Noxa expression. In addition, Noxa upregulation was reported to be associated with the proapoptotic effects of several polyphenols such as resveratrol, phenoxodiol or tetramethoxystilbene as well as the flavonoid isoliquiritigenin (37-40). Therefore, such a family of natural specific Noxa upregulators might be useful for the development of novel combining therapeutic protocols in CLL. As regards hyperforin, a derivative called aristoforin has been described with improved stability and solubility as well as better biological activity (41). By contrast, the biochemical structure of M2Yn is not yet fully defined. The natural extract consists of a mixture of several components retaining its biological activity upon protein depletion. Several flavonoids were found among these components, and experiments are in progress in order to identify which of them is (are) responsible for the proapoptotic activity of M2Yn. The complete chemical structure of M2Yn will allow to determine whether this activity may also be attributed to other types of compounds such as phloroglucinols or terpenoids.

Data from the present study confirm that the BH3-only protein Noxa is an attractive target for CLL therapy. The concept of BH3 mimetics has recently emerged $(29,30)$, and several $\mathrm{BH} 3$ mimetics displaying the capacity to antagonize the antiapoptotic $\mathrm{Bcl}-2 / \mathrm{Bcl}-\mathrm{xL} / \mathrm{Bcl}-\mathrm{w}$ proteins have been described such as ABT-737 (42). As regards the crucial role of Mcl-1 in CLL, the use of Noxa mimetics should be of great interest. In connection with this, the clinical developments of pan-BH3 mimetics which efficiently mimic Noxa and/or target Mcl-1 (2), such as Obatoclax/GX15-070, Gossypol/ AT-101 or the peptide 072RB, seem promising (8,43-45).

\section{Acknowledgements}

We are indebted to Professor Amu Therwath for critical reading of the manuscript. We also thank Dr Zohar Mishal for mass spectrometry analysis, and Mrs. Fanny Fava and Julia Prado for technical assistance.

\section{References}

1. Ghia P, Ferreri AJM, and Caligaris-Cappio F: Chronic lymphocytic leukemia. Crit Rev Oncol Hematol 64: 234-246, 2007.

2. Pleyer L, Egle A, Hartmann TN and Greil R: Molecular and cellular mechanisms of CLL: novel therapeutic approaches. Nat Rev Clin Oncol 6: 405-418, 2009.

3. Tam CS and Keating MJ: Chemoimmunotherapy of chronic lymphocytic leukemia. Nat Rev Clin Oncol 7: 521-532, 2010.

4. Kolb JP, Kern C, Quiney C, Roman V and Billard C: Re-establishment of a normal apoptotic process as a therapeutic approach in B-CLL. Curr Drug Targets Cardiovasc Hematol Disord 3: 261-286, 2003.

5. Byrd JC, Shinn C, Waselenko JK, et al: Flavopiridol induces apoptosis in chronic lymphocytic leukemia cells via activation of caspase- 3 without evidence of bcl- 2 modulation or dependence on functional p53. Blood 92: 3804-3816, 1998. 
6. Lucas DM, Edwards RB, Lozanski G, et al: The novel plantderived agent silvestrol has B-cell selective activity in chronic lymphocytic leukemia and acute lymphoblastic leukemia in vitro and in vivo. Blood 113: 4656-4666, 2009.

7. Hahntow IN, Schneller F, Oelsner M, et al: Cyclin-dependent kinases inhibitor Roscovitine induces apoptosis in chronic lymphocytic leukemia. Leukemia 18: 747-755, 2004.

8. Balakrishnan K, Wierda WG, Keating MJ and Gandhi V: Gossypol, a BH3 mimetic, induces apoptosis in chronic lymphocytic leukemia cells. Blood 112: 1971-1980, 2008.

9. Roman V, Billard C, Kern C, et al: Analysis of resveratrolinduced apoptosis in human B-cell chronic leukaemia. Br J Haematol 117: 1-10, 2002.

10. Billard C, Menasria F, Quiney C, Faussat, AM, Finet JP, Combes S and Kolb JP: 4-arylcoumarin analogues of combretastatins stimulate the apoptosis of leukemic cells from chronic lymphocytic leukemia patients. Exp Hematol 36: 1625-1633, 2008.

11. Quiney C, Billard C, Faussat AM, et al: Pro-apoptotic properties of hyperforin in leukemic cells from patients with chronic lymphocytic leukemia. Leukemia 20: 491-497, 2006.

12. Kitada S, Zapata JM, Andreeff M and Reed JC: Protein kinase inhibitor flavopiridol and 7-hydroxystaurosporine down-regulate antiapoptosis proteins in B-cell chronic lymphocytic leukemia. Blood 96: 393-397, 2000

13. Chen R, Keating MJ, Gandhi V and Plunkett W: Transcription inhibition by flavopiridol: mechanism of chronic lymphocytic leukemia cell death. Blood 106: 2513-2519, 2005.

14. Gandhi V, Balakrishnan K and Chen LS: Mcl-1: the 1 in CLL. Blood 112: 3538-3540, 2008.

15. Zaher M, Akrout I, Mirshahi M, Kolb JP and Billard C: Noxa upregulation is associated with apoptosis of chronic lymphocytic leukemia cells induced by hyperforin but not flavopiridol. Leukemia 23: 594-596, 2009.

16. Willis SN, Chen L, Dewson G, Wei A, Naik E, Fletcher JI, Adams JM and Huang DCS: Proapoptotic Bak is sequestered by Mcl-1 and Bcl-xL, but not Bcl-2, until displaced by BH3-only proteins. Genes Dev 19: 1294-1305, 2005.

17. Qin JZ, Ziffra J, Stennett L, et al: Proteasome inhibitors trigger Noxa-mediated apoptosis in melanoma and myeloma cells. Cancer Res 65: 6282-6293, 2005.

18. Inoue S, Riley J Gant TW, Dyer MJS and Cohen GM: Apoptosis induced by histone deacetylase inhibitors in leukemic cells is mediated by Bim and Noxa. Leukemia 21: 1773-1782, 2007.

19. Flinn IW, Byrd JC, Bartlett N, et al: Flavopiridol administered as a 24-hour continuous infusion in chronic lymphocytic leukemia lacks clinical activity. Leuk Res 29: 1253-1257, 2005.

20. Faderl S, Rai K, Gribben J, et al: Phase II study of single-agent bortezomib for the treatment of patients with fludarabine-refractory B-cell chronic lymphocytic leukemia. Cancer 107: 916-924, 2006.

21. Byrd JC, Marcucci G, Parthum MR, et al: A phase 1 and pharmacodynamic study of depsipeptide (FK228) in chronic lymphocytic leukemia and acute myeloid leukemia. Blood 105: 959-967, 2005

22. Hallek M, Cheson BD, Catovsky D, et al: Guidelines for the diagnosis and treatment of chronic lymphocytic leukemia: a report from the International Workshop on Chronic Lymphocytic Leukemia (IWCLL) updating the National Cancer Institute-Working Group (NCI-WG) 1996 guidelines. Blood 111: 5446-5456, 2008.

23. Billard C, Kern C, Tang R, Ajchenbaum-Cymbalista F and Kolb JP: Flavopiridol downregulates the expression of both the inducible NO synthase and $\mathrm{p} 27^{\mathrm{kipl}}$ in malignant cells from B-cell chronic lymphocytic leukemia. Leukemia 17: 2345-2443, 2003.

24. Fourneron JD and Naït-Si Y: Effect of eluent pH on HPLC/UV analysis of hyperforin. Phytochem Anal 17: 71-77, 2006.

25. Kern C, Cornuel J, Billard C, et al: Involvement of BAFF and APRIL in the resistance to apoptosis of B-CLL through an autocrine pathway. Blood 103: 679-688, 2004.

26. Moravec RA, O'Brien MA, Daily WJ, Scurria MA, Bernad L and Riss TL: Cell-based bioluminescent assays for all three proteasome activities in a homogeneous format. Anal Biochem 387: 294-302, 2009.
27. Dai Y, De Sano J, Tang W, et al: Natural proteasome inhibitor celastrol suppresses androgen-independent prostate cancer progression by modulating apoptotic proteins and NF-kappaB. PLoS One 5: e14153, 2010.

28. Chen D, Daniel KG, Chen MS, Kuhn DJ, Landis-Piwowar KR and Ping Dou Q: Dietary flavonoids as proteasome inhibitors and apoptosis inducers in human leukemia cells. Biochem Pharmacol 69: 1421-1432, 2005.

29. Packham G and Stevenson FK: Bodyguards and assassins: Bcl-2 family proteins and apoptosis control in chronic lymphocytic leukemia. Immunology 114: 441-449, 2005.

30. Awan FT, Kay NE, Davies ME, et al: Mcl-1 expression predicts progression-free survival in chronic lymphocytic leukemia patients treated with pentostatin, cyclophosphomide, and rituximab. Blood 133: 535-537, 2009.

31. Hussain SR, Cheney CM, Johnson AJ, Lin TS, Grever MR, Caligiuri MA, Lucas DM and Byrd JC: Mcl-1 is a relevant therapeutic target in acute and chronic lymphoid malignancies: Downregulation enhances rituximab-mediated apoptosis and complement-dependent cytotoxicity. Clin Cancer Res 13: 144-150, 2007.

32. Smit L, Hallaert DYH, Spijker R, et al: Differential Noxa/Mcl-1 balance in peripheral versus lymph node chronic lymphocytic leukemia cells correlates with survival capacity. Blood 109: 1660-1668, 2007

33. Blagoskonny MV: Flavopiridol, an inhibitor of transcription. Implications, problems ans solutions. Cell Cycle 3: 1537-1542, 2004.

34. Nencioni A, Hua F, Dillon CP, et al: Evidence for a protective role of Mcl-1 in proteasome inhibitor-induced apoptosis. Blood 105: 3255-3262, 2005

35. Qin JZ, Xin H, Sitailo LA, Denning MF and Nickoloff BJ: Enhanced killing of melanoma cells by simultaneously targeting Mcl-1 and NOXA. Cancer Res 66: 9636-9645, 2006.

36. Kress CL, Konopleva M, Martinez-Garcia V, et al: Triterpenoids display single agent anti-tumor activity in a transgenic mouse model of chronic lymphocytic leukemia and small B cell lymphoma. PLos One 2: e559, 2007.

37. Shankar S, Singh G and Srivastava RK: Chemoprevention by resveratrol: molecular mechanisms and therapeutic potential. Front Biosc 1: 4839-4854, 2007.

38. Yu F, Watts RN, Zhang XD, Borrow JM and Hersey P: Involvement of $\mathrm{BH} 3$-only proapoptotic proteins in mitochondrial-dependent Phenoxodiol-induced apoptosis of human melanoma cells. Anticancer Drugs 17: 1151-1161, 2006.

39. Aiyar SE, Park H, Aldo PB, et al: TMS, a chemically modified herbal derivative of Resveratrol, induces cell death by targeting Bax. Breast Cancer Res Treat 124: 265-277, 2010.

40. Hsu YL, Kuo PL and Lin CC: Isoliquiritigenin induces apoptosis and cell cycle arrest through p53-dependent pathway in Hep G2 cells. Life Sci 3: 279-292, 2005.

41. Rothley M, Schmid A, Thiele W, et al: Hyperforin and aristoforin inhibit lymphatic endothelial cell proliferation in vitro and suppress tumor-induced lymphangiogenesis in vivo. Int J Cancer 125: 32-42, 2009

42. Van Delft MF, Wei AH, Mason KD, et al: The BH3 mimetic ABT-737 targets selective Bcl-2 proteins and efficiently induces apoptosis via Bak/Bax if Mcl-1 is neutralized. Cancer Cell 10: 389-399, 2006.

43. Nguyen M, Marcellus RC, Roulston A, et al: Small molecule obatoclax (GX15-070) antagonizes MCL-1 and overcomes MCL-1-mediated resistance to apoptosis. Proc Natl Acad Sci USA 104: 19512-19517, 2007.

44. Ghiotto F, Fais F, Tenca C, et al: Apoptosis of B-cell chronic lymphocytic leukemia cells induced by a novel BH3 peptidomimetic. Cancer Biol Ther 8: 263-271, 2009.

45. O'Brien SM, Claxton DF, Crump M, et al: Phase I study of obatoclax mesylate (GX15-070), a small molecule pan-Bcl-2 family antagonist, in patients with advanced chronic lymphocytic leukemia. Blood 113: 299-305, 2009. 